\title{
Jumpstarting Electron Motion in Molecules
}

\author{
Ultrashort pulses of $x$ rays swap electrons around in molecules of nitric
}

oxide, an important first step to tracking charge motion in molecules.

\section{By Christopher Crockett}

\rceil o observe the dance of electrons during a chemical reaction, researchers can illuminate the system with an ultrashort pulse of $x$ rays, which reveals charge dynamics on an attosecond timescale. While such blasts of $x$ rays have been used to unveil these electronic shifts around single atoms, researchers had not yet done so for molecules. In a first step toward this goal, Jordan O'Neal of Stanford University, California, and colleagues have successfully used $x$ rays to initiate electron motion in molecules of nitric oxide [1]. The next step is tracking the electrons' motion, something that could allow researchers to learn more about the intricate transfer of charge that accompanies chemical reactions.

In their experiments, the team used an ultrashort x-ray pulse to rapidly shuffle electrons around the molecule's oxygen atom, knocking the electrons' arrangement out of equilibrium. The $x$-ray pulse excited an electron from the innermost oxygen orbital to a vacant valence orbital. Normally, this electron would return to its home orbital almost immediately. But a

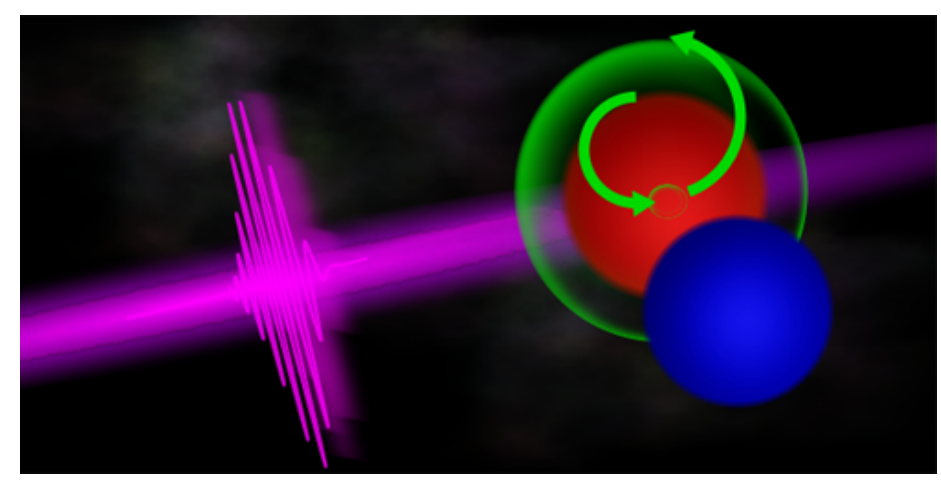

Credit: J. P. Cryan and J. T. O'Neal/SLAC National Accelerator Laboratory second interaction with the same $\mathrm{x}$-ray pulse knocked an electron from a different valence orbital down to the core orbital, plugging the hole left behind by the first electron and putting the molecule in a relatively stable excited state.

Getting the molecule into this state is important, the team says, because a change in electron configuration-which is what they initiated here-is the first step in any chemical reaction. Tracking how the process unfolds subsequently-the way the excited electron spreads around the entire molecule, for example-is the researchers' aim for upcoming experiments.

Christopher Crockett is a freelance writer based in Arlington, Virginia.

\section{REFERENCES}

1. J. T. O’Neal et al., "Electronic population transfer via impulsive stimulated x-ray Raman scattering with attosecond soft-x-ray pulses," Phys. Rev. Lett. 125, 073203 (2020). 